\title{
Ébauche d'une caractérisation de l'anglais de l'histoire
}

Jean-Louis Trouillon

\section{(2) OpenEdition}

1 Journals

Édition électronique

URL : http://journals.openedition.org/asp/97

DOI : $10.4000 /$ asp. 97

ISBN : 978-2-8218-0410-4

ISSN : 2108-6354

\section{Éditeur}

Groupe d'étude et de recherche en anglais de spécialité

Édition imprimée

Date de publication : 1 novembre 2009

Pagination : 5-27

ISSN : 1246-8185

\section{Référence électronique}

Jean-Louis Trouillon, «Ébauche d'une caractérisation de l'anglais de l'histoire », ASp [En ligne], 56

2009, mis en ligne le 25 mai 2012, consulté le 06 novembre 2020. URL : http://

journals.openedition.org/asp/97 ; DOI : https://doi.org/10.4000/asp.97

Ce document a été généré automatiquement le 6 novembre 2020.

Tous droits réservés 


\title{
Ébauche d'une caractérisation de l'anglais de l'histoire
}

\author{
Jean-Louis Trouillon
}

1 Le titre même de cette étude pourrait faire l'objet d'un questionnement. Si les raisons d'un choix entre « anglais historique » et «anglais de l'histoire » ne présentent guère d'intérêt puisque dans notre secteur LANSAD l'on trouve aussi bien «anglais juridique » que "anglais du droit », même si d'autres disciplines préfèrent l'une ou l'autre construction (c'est ainsi par exemple que «anglais scientifique "s'oppose à « anglais de l'économie » alors que l'on trouvera très rarement « anglais des sciences » ou, pour des raisons évidentes, « anglais économique »), la forte présence de l'historien que l'on remarque dans les écrits, ainsi qu'il sera montré infra, aurait sans doute permis de proposer aussi «anglais de l'historien » voire « anglais des historiens ». Une autre possibilité, enfin, aurait été de plagier Deleplace (2006) et son « discours historien » et de proposer ainsi l'appellation néologique «anglais historien». La sagesse prévaut toutefois dans un premier temps et les normes habituelles de dénomination ont donc été conservées.

2 Par ailleurs, le terme « ébauche » peut faire sourire du fait de son apparente naïveté : déjà au $\mathrm{II}^{\mathrm{e}}$ siècle avant notre ère, en effet, il y avait interrogation sur l'écriture de l'histoire, et c'est ainsi que l'on peut relever sous la plume ${ }^{1}$ de Lucien de Samosate dans le chapitre au titre normatif Comment il faut écrire l'histoire:

Parlons d'abord de ce que doit éviter l'historien, de ce dont il faut qu'il ait grand soin de s'abstenir ; ensuite nous dirons ce qu'il a à faire pour ne jamais s'écarter de la ligne droite et suivre toujours le vrai chemin; de quelle manière il doit commencer, à quel ordre il doit s'astreindre dans son ouvrage, quelle est la mesure de chaque partie, ce qu'il faut taire, sur quoi il faut insister, ce qu'il vaut mieux esquisser d'un trait rapide, avec quel soin tout doit être exprimé et enchaîné : tous ces préceptes, et autres semblables, viendront en second lieu. Dès à présent, nous allons dire quels sont les défauts ordinaires des mauvais historiens. Ceux qui sont communs à tous les genres de style, et qui tiennent à la langue, à l'arrangement des mots, aux pensées, toutes les maladresses enfin de cette nature seraient trop longues à exposer ici, et en dehors de mon sujet: les fautes, en effet, qui se 
commettent contre la langue et le style sont communes à tous les genres. (1912 :

1-6) dans la citation qui vient d'être rappelée, comme étant «l'arrangement des mots» puis, quelques lignes plus loin, « la langue et le style». Cette étude s'attachera donc à aborder le discours de l'histoire selon les méthodes proposées par la communauté LANSAD.

\section{La terminologie}

7 Il est à noter que Lucien mentionne l'arrangement des mots et non les mots euxmêmes, anticipant ainsi une première constatation du chercheur contemporain à savoir qu'au premier abord il ne semble pas exister de terminologie spécifique à l'histoire en tant que discipline, contrairement à ce qui existe dans l'ensemble des domaines étudiés à ce jour.

8 La mise en écrit et les choix lexicaux qui en découlent interpellent pourtant l'historien: «comment analyser le passé dans un charabia incompréhensible et inadéquat?» (Prochasson 2008). Mais dans notre domaine LANSAD, dès qu'il est question de lexique, l'on est immédiatement amené à se pencher plus particulièrement sur la terminologie qui est l'un de nos axes d'approche privilégiés, que ce soit au niveau de la recherche ou au niveau de l'enseignement de la langue pour le spécialiste ou futur spécialiste du domaine.

9 L'histoire présente néanmoins un cas original par rapport à des disciplines comme les sciences physiques, l'économie ou le droit : "Tout est objet d'histoire dans les manifestations humaines, du public à l'intime, que ce soit à travers les discours, les pratiques ou les représentations » (Guereña 2003 : paragraphe 19).

10 L'histoire, telle qu'elle nous apparait, n'existe, en effet, que parce qu'elle nous parle de faits humains, sociaux, techniques, scientifiques, artistiques ou culturels, éventuellement en relation avec le milieu géographique naturel, si l'on pense par exemple à ce qu'il est convenu d'appeler, à la suite des travaux de Le Roy Ladurie, l'histoire du climat. Dans la description et l'analyse qu'elle fait de ces phénomènes on 
retrouvera la terminologie propre à chacune de ces disciplines scientifiques, artistiques, culturelles ou autres.

11 Quant aux techniques utilisées par les historiens lors de l'activité de recherche proprement dite, c'est-à-dire lors du temps précédant la diffusion de leurs découvertes, elles possèdent également leur propre terminologie. Le paléographe emprunte la terminologie du linguiste et du philologue, le classement des archives est dû aux archivistes et, sans vouloir multiplier les exemples et citer la totalité de ces «sciences annexes » comme la diplomatique ou l'épigraphie pour ne rappeler que les plus fréquentes, il est évident que l'outil le plus récent, l'informatique, avec les traitements de texte, d'image ou de statistiques qu'elle permet, possède un discours qui lui est propre.

De fait, lorsque l'on étudie, soit à la simple lecture soit au moyen d'outils statistiques, des documents rédigés par des historiens, ce qui ressort immédiatement est l'absence quasi totale de termes créés par les historiens pour leur propre discipline, c'est-à-dire des termes proposés a posteriori pour décrire ou expliquer des phénomènes antérieurs. Un rare exemple, pour illustrer ce propos, pourrait être, selon nous, « thalassocratie " que Le Dictionnaire universel de la langue française de Pierre Boiste, publié en 1828, attribue à Francesco Bianchini pour caractériser la Crète antique, probablement dans son Histoire universelle publiée à Rome en 1697 mais dont on trouve en fait une première occurrence dans la Géographie de Strabon. Il s'agit donc bien d'un véritable terme puisque créé (sur les modèles déjà existants de démocratie, théocratie et autres) près de deux millénaires plus tard pour décrire la réalité politique crétoise d'alors.

Des historiens consultés sur ce point ont corroboré notre observation. Voici une synthèse de ce qui ressort d'échanges avec ces professionnels :

L'histoire se donne pour objectif de tenter de reconstituer les faits d'une part, et de leur donner une cohérence, d'expliquer les articulations qui amènent à la succession d'événements. Pour cela, il faut effectivement rendre compréhensibles les faits en 'traduisant' en quelque sorte les phénomènes du passé aux hommes de notre temps. La tradition 'littéraire' de l'histoire comme discipline fait que la 'mise en récit' n'utilise pas de terminologie particulière. [...] L'histoire est fortement emprunteuse: elle utilise les concepts, la terminologie des 'champs' qu'elle se propose d'analyser. (Marty 2009) ${ }^{2}$

\section{Comment on écrit l'histoire (Veyne 1970)}

14 Puisque la terminologie ne sera pas une approche envisageable et que les historiens ont eux-mêmes réfléchi à l'écriture de leur discipline, il est possible d'aborder notre étude grâce aux travaux de ceux qui se sont demandé "comment on écrit l'histoire ». Un certain nombre d'indices sont alors accessibles et ils ouvrent une perspective d'approche :

L'histoire s'écrit sous le regard de l'autre et cela implique toute une série d'égards : il y a comme cela une politesse de l'histoire, avec ses règles et son étiquette, comme d'adopter le bon ton, de justifier ses choix et de fournir des explications là où cela paraît nécessaire, de mettre en garde contre les conclusions hâtives, toutes choses qui font partie du rituel de l'interprétation. (Quéré 2001 : 131)

Une recherche bibliographique permet, dans un premier temps, de trouver de nombreux conseils de rédaction qui s'adressent généralement aux jeunes étudiants d'universités anglophones désireux d'entreprendre des études supérieures d'histoire. Il 
s'agit là de conseils d'ordre méthodologique semblables à tous ceux qui sont donnés, de par le monde, aux étudiants en lettres et sciences humaines.

Leur lecture, pour intéressante qu'elle soit, ne répond cependant pas entièrement à nos interrogations dans la mesure où il s'agit en effet de normes proposées à des étudiants pour la rédaction d'essais, alors que la communauté LANSAD choisit traditionnellement ses sujets d'étude parmi les productions de spécialistes de la discipline et non celles des apprenants de ladite discipline ${ }^{3}$.

Notre hypothèse étant toutefois que les historiens professionnels ont d'abord été étudiants et auront donc probablement tendance à reproduire dans leurs travaux les normes qui leur ont été enseignées, normes qu'ils enseignent très certainement euxmêmes d'ailleurs, il apparaît logique de commencer notre étude par l'examen de ces normes de rédaction telles qu'elles sont indiquées par un historien universitaire dans un cours de méthodologie à destination de ses étudiants. L'analyse ultérieure des véritables productions de spécialistes retenues dans notre corpus permettra d'ailleurs de vérifier dans quelle mesure ces conseils aux apprenants sont réutilisés par les professionnels. Il faudra également garder en mémoire la différence de visée entre les différentes productions :

Le professeur est poussé vers la vulgarisation destinée au «grand public» (étudiant ou non), alors que le spécialiste s'exile des circuits de la consommation. La production historique s'en trouve partagée entre l'œuvre littéraire de qui «fait autorité » et l'ésotérisme scientifique de "qui fait de la recherche». (de Certeau 1975 : 90)

Le document proposé pour cet exemple provient de la Southern Oregon University (Carney 1996); il est représentatif de tous ceux que l'on peut consulter sur le même sujet.

Dans la mesure où "a history paper is an essay ", l'auteur définit tout d'abord ce qu'est un essai et quel est l'objectif que l'essai vise à atteindre : «Systematic argumentation [...] is the point of an essay. Essays express the educated opinion of their writers ». La structure type de l'essai est alors rappelée :

The introduction can be conceptualized as a triangle tipped on its side. It begins broadly and comes to a point. This point is your thesis. A thesis is a concise statement of your opinion, and has been called "essence of essay." Connected to the triangle at the point is the main body of the essay. This contains your argument and your evidence. It flows naturally out of your thesis, and proceeds point-by-point to the conclusion. The conclusion begins where your last point left off. It then widens back out to the more general. This wide-narrow-wide structure expresses the purpose of the essay - present an opinion on a small, narrow topic and relate it to the larger world, to life in general. If you cannot relate a topic to larger issues, it is not worth writing about.

L'introduction, le développement et la conclusion sont ensuite décrits en détail (cf. Annexe). L'auteur donne, dans la dernière partie de son document en ligne, un certain nombre de conseils d'ordre stylistique :

Avoid passive voice, eliminate unnecessary words, be authoritative, make the paragraph the basic unit of your writing, keep to one tense, vary your choice of words, never use 'there' - ever, use 'that' very sparingly, vary the length of your sentences, make sure pronoun references agree in type and number, do not use redundancies. 
Il est possible de compléter ces conseils grâce à des données relevées dans le Dartmouth Writing Program : "Use the past tense, avoid the subjective I, write as dispassionately as you can » (Gogsik 2005).

Nous verrons infra que ces judicieuses recommandations, que l'on pourrait d'ailleurs proposer à tous les étudiants en lettres et sciences humaines de culture occidentale, et pas seulement aux futurs historiens, ne sont pas toujours systématiquement suivies par les historiens professionnels.

\section{Le choix du corpus}

3 Le corpus retenu est dû à ces universitaires ou chercheurs qui viennent d'être appelés historiens professionnels, "ceux qui sont payés pour faire ce travail» (Prochasson 2008). Même si l'on assiste à « a trend for history to be seen no longer as the preserve of the professional 》 (Coffin 2006 : 3) et bien que certains éminents historiens acceptent parfois cette intrusion ainsi que Fernand Braudel l'aurait déclaré à Alain Minc $(2008)^{4}$, nous ne nous attacherons, pour cette première analyse tout au moins, qu'au seul discours professionnel et non à l'ensemble du discours disciplinaire (Petit $2004: 8$ ).

À l'exception de l'ouvrage collectif de Hobsbawm (1983), les documents retenus pour le corpus d'étude sont en majorité des articles publiés dans des revues de recherche universitaires, dont on sait qu'elles s'adressent en principe à un public d'historiens professionnels, et ce contrairement aux ouvrages disponibles en librairie ou en bibliothèque qui ambitionnent de toucher un public cette fois élargi aux amateurs éclairés. L'écriture de ces derniers ouvrages pourrait en effet porter des traces de cet objectif de diffusion, si l'on en croit les propos de Sir John Plumb rapportés par son élève Schama: "he taught us that writing was not just an auxiliary to research, he wanted people outside the academy to read history, he wanted it to be entertaining ".

25 Les auteurs sont tous des locuteurs anglophones natifs, britanniques, américains et australiens, certains vivent et exercent en France et ont publié en anglais dans des revues françaises. L'échantillon, sans être statistiquement représentatif, permet néanmoins de ne pas limiter notre étude à une seule origine géographique des auteurs.

26 Ces derniers appartiennent à des tranches d'âge différentes et sont soit illustres du fait de leur ancienneté et leur qualité (Hobsbawm et Trevor-Roper), soit moins connus du public de spécialistes. L'on verra plus tard que ce paramètre de célébrité peut justifier certains écarts par rapport aux normes communément admises.

27 Les sujets traités sont très variés, sociaux, militaires ou religieux par exemple.

Le dernier critère de sélection était la date de publication, choisie au cours de ces dernières décennies afin d'éviter l'influence de changements diachroniques éventuels.

Enfin, quinze des articles sont ceux d'auteurs individuels qui se répartissent en six hommes et neuf femmes. Le dernier article est dû à trois co-auteurs, deux femmes et un homme. Ces deux caractéristiques seront commentées infra.

Puisque la terminologie ne semble pas être un axe de recherche prometteur, nous poserons comme hypothèse de travail que, l'article de recherche en histoire appartenant bien à un genre tel qu'il fut défini et analysé par Swales (1990), nous nous attacherons à étudier les phénomènes récurrents qui sous-tendent sa structure et, plus 
généralement, à analyser l'ensemble de la démarche et des modes de présentation adoptés par les auteurs.

\section{La présentation de l'article}

31 À une exception près (McCullough et al. 2006), chaque article du corpus a été écrit par une seule personne, ce qui est certainement un trait caractéristique de la recherche en sciences humaines en général, ainsi qu'il nous est facile d'en juger à partir de nos diverses revues professionnelles. L'histoire ne déroge donc pas à cette coutume.

McCullough, Heat et Fields appartiennent à trois universités différentes (Cambridge, Royaume-Uni ; University of Utah et Indiana State University, États-Unis) et surtout exercent dans trois départements bien distincts : Anthropology, Geography and Geology et History. Le sujet retenu, que l'on peut résumer par la première phrase de l'abstract, justifie cette collaboration entre différents experts: "Kin selection, an aspect of evolutionary theory, argues that, all else equal, individuals should give preferential treatment to relatives, based on the degree of relatedness ". Il s'agit en effet dans la thèse proposée de synthétiser des données provenant de diverses spécialités.

Le chercheur LANSAD qui a l'habitude d'étudier de nombreux articles de recherche dans différentes disciplines sera immédiatement frappé par une lacune fréquente. Bien rares en effet sont les articles accompagnés d'un abstract et de mots clés. Au sein de notre corpus, on ne relève leur présence systématique que dans les revues appartenant au groupe Elsevier, qu'il s'agisse du Journal of Medieval History ou de The History of the Family.

Le rapport entre la présence ou l'absence de ces deux éléments n'a pas été étudié, ce rapport aurait été dépourvu de valeur statistique sur un échantillon aussi limité, et par ailleurs l'abondance des revues scientifiques historiques, qu'il s'agisse de publications traditionnelles écrites ou de publications électroniques disponibles sur la toile, n'a en effet pas permis une réelle étude statistique dans le cadre de notre étude. Cette absence est tout de même suffisamment fréquente pour devoir être signalée.

Les abstracts, lorsqu'ils existent, correspondent assez largement à ce qui est couramment admis pour l'abstract scientifique ; il n'y a aucune originalité particulière à ce niveau, même si l'on peut évidemment constater quelques écarts d'un auteur à l'autre :

- présentation du sujet (This article studies... ou This paper examines...),

- rappel des connaissances actuelles (Using these sources, historians have concluded that...),

- originalité de l'article (This article reaches different conclusions than had previously been suggested ou it will also contest...),

- justification de la démarche (It argues that... ou We show that).

Comme pour tout article de recherche, les mots clés, s'ils sont considérés séparément et dans le désordre, apparaissent bien généraux (Youth, Adolescents, Darwinian evolution, Australia, England, Clergy, Warfare, Migration...). Ils sont donc difficilement utilisables pour une recherche de documentation qui ne procèderait que par mots clés individuels. Ils ne prennent évidemment tout leur sens qu'en raison de leur combinaison, ainsi que le montrent les trois exemples suivants qui respectent l'ordre original de présentation des mots :

-Servants ; Life cycle; Illegitimacy ; Migration; Australia (Swain 2005); 
- Clergy ; Illegitimacy ; Dispensation ; Canon law ; England (Wertheimer 2005) ;

- Edward I ; Pastoral care ; Military chaplains; Warfare ; Royal administration (Bachrach 2003).

\section{La structure de l'article}

Les intertitres ou titres de paragraphes sont rares, leur présence ou absence semble dépendre aussi bien de la politique éditoriale de la revue que du choix personnel de l'auteur. L'absence d'intitulés n'empêche évidemment pas d'avoir affaire à des documents généralement fortement structurés. L'on retrouve ainsi assez systématiquement, mais pas toujours dans cet ordre d'apparition, les points suivants.

En introduction les objectifs poursuivis par l'auteur (This article will analyze.... / It will examine... / The article will provide a basis for... / This article investigates... / This article seeks to examine... / it will argue that... / it will also contest...).

Vient ensuite la méthode utilisée par l'auteur (...it will use... / Any analysis must also take into consideration... / We need to look at...).

L'appel aux sources primaires, matériau de base de l'historien, est évidemment présent (The available figures on... / According to... / Some idea of [...] can be gleaned from... /...compiled from a range of newspapers). Ces sources peuvent d'ailleurs être commentées et c'est alors généralement soit pour regretter leur insuffisance (...in the absence of detailed census data... / ...the available census data seldom provide...) soit pour souligner leur manque de fiabilité (...the reliability of data on [...] is often compromised by...).

41 Comme dans tout article de recherche il y a reconnaissance du travail accompli par les prédécesseurs (...as one recent study has termed it... / a considerable amount of research carried out... / One feminine authority on the subject has written that.../ Some useful work has been undertaken on... / Most historians have assumed that... / As Rose's work has indicated.... / As Palmer has argued... / According to Higgs... / We are told...). Ce point est développé infra.

Il y a évidemment justification de la niche de recherche en montrant l'intérêt d'une nouvelle approche (...they are well worth examining for the light they throw).

Cette justification fait généralement ressortir une lacune dans les travaux antérieurs (More work needs to be done on... / Comparatively little work has been carried out... / Despite recent research relatively little is known about... / There has been no attempt, to date, to analyze in depth... / There has been one significant lacuna... / Historical writing shied away from... / Therefore in this study an effort is made to... / These works are well worth unearthing from obscurity).

tude peut aussi être annoncée en opposition à l'historiographie traditionnelle $\mathrm{du}$ domaine (There are a number of methodological problems in the way that [...] have been studied / While Higman's research would suggest that... / More than a century later it is perhaps difficult to appreciate why... / Some recent historians have been inclined to emphasize... / We had been led to believe by the earliest research...). Plus rarement, l'auteur s'appuie sur une incertitude quant aux conclusions tirées des travaux antérieurs (There is also disagreement among historians about...).

Le développement de l'idée originale de l'auteur s'effectue le plus souvent selon les règles habituelles de prudence dans l'énoncé de nouvelles propositions (...would probably have been... / This tendency can be seen partly as...), mais peut parfois assumer franchement des certitudes (...there is no doubt at all... / ...it is equally certain however ... 
/ ...we shall never know... / We can clearly see here... / ...the following statement clearly indicates...).

La conclusion s'inscrit généralement dans la tradition universitaire, c'est-à-dire qu'elle rappelle d'abord les découvertes de l'auteur (As the preceding survey clearly indicates... / The evidence indicates that... / The evidence in this article would suggest that...) et élargit ensuite le champ d'investigation vers un travail ultérieur (Closer examination of [...] may reveal... / A close examination of [...] might reveal similar paradoxes) ${ }^{5}$.

Ces derniers may et might montrent bien la prudence dont s'entoure l'auteur qui n'hésite pas à avouer les limites de son étude (It is not clear... / Unfortunately for this study the [...] is not accurately recorded, but the data suggest...).

Cette prudence du chercheur (We need to be cautious in handling evidence relating to...) est justifiée par la nature même de l'article de recherche dont l'objectif est de proposer une thèse en faveur de laquelle l'historien développe une argumentation, en prévoyant les objections éventuelles qui ne manqueront pas de lui être adressées (...it may be argued that...).

Le raisonnement (The inference to be drawn is...) est souligné par un nombre élevé de therefore que l'on retrouve systématiquement chez chaque auteur et pratiquement dans chaque paragraphe de la démonstration. Consequently, hence, subsequently, thereby, thus sont également très fréquents, renforçant la logique interne du discours.

La démonstration s'appuie d'autre part sur la présence des connecteurs habituels permettant l'introduction de nouvelles idées ou suggestions : however, though, although, instead, in spite of, on the one hand / on the other hand se retrouvent fréquemment tout au long du texte, et ce pour chacun des éléments constituants du corpus.

51 Les conseils méthodologiques relatifs à l'organisation de la démonstration rappelés supra sont donc suivis par les professionnels.

\section{L'implicite}

52 L'implicite fait partie des caractéristiques du discours scientifique en général, quel que soit le domaine d'application. Les destinataires partagent en commun avec l'auteur un ensemble de connaissances du domaine, ce qui évite à l'énonciateur de rappeler ou de définir systématiquement l'ensemble des données auxquelles il fait appel.

Cette caractéristique se retrouve bien évidemment dans notre contexte, ce qui exige du lecteur cultivé, mais non spécialiste de l'époque, qu'il soit parfois amené à rechercher par ailleurs des informations complémentaires.

Nous pouvons citer en exemple le titre Illegitimate birth and the English Clergy, 1198-1348 (Wertheimer 2005). Ni l'abstract ni l'article lui-même ne justifient le choix de ces deux dates qui sont donc supposées connues de tout médiéviste. Certes, les médiévistes reconnaîtront sans hésitation dans la date de 1348 la grande année de propagation de la peste noire qui correspond à la fin de ce que Le Goff a nommé Moyen-Âge central, mais ces mêmes médiévistes pourraient être pardonnés s'ils ne se souvenaient ex abrupto que 1198 correspond au début du pontificat d'Innocent III, bien connu de tous pour sa lutte contre les Cathares ${ }^{6}$ et qui s'attacha parallèlement à reprendre en main le clergé jusqu'alors souvent fortement critiqué pour le laxisme de ses mœurs. 


\section{« Le texte feuilleté » (Prost 1996)}

55 Tout document de recherche s'appuie nécessairement, dans notre tradition universitaire, sur des travaux antérieurs, qu'ils portent directement sur le même sujet ou, s'ils en diffèrent, qu'ils soient utilisés à des fins méthodologiques. Les observations et remarques personnelles du chercheur sont en principe également mentionnées et classées.

L'histoire obéit évidemment à ces normes, tout en respectant sa propre démarche :

Le texte historique se déploie sur deux niveaux distincts, qu'il entremêle pourtant sans cesse.

Le premier niveau est celui du discours de l'historien: son intrigue et son argumentation. Ce texte est continu, structuré, maîtrisé. Il dit le déroulement et la signification de l'histoire, établit les faits, discute les explications possibles.

Sans cesse, pourtant, ce discours s'interrompt plus ou moins brièvement, pour des références, des citations. Dans le texte historique apparaissent ainsi, épisodiquement, des fragments d'autres textes, empruntés parfois à d'autres historiens, mais plus souvent à des documents d'époque, chroniques ou témoignages. Le texte de l'historien comprend ainsi en un double sens, matériel et interprétatif, la parole d'un autre, de plusieurs autres. Mais c'est une parole découpée, démembrée, déconstruite et reconstruite par l'historien qui la réemploie, à la place qu'il a lui même choisie en fonction des nécessités de son propre discours. (Prost 1996)

57 Afin d'étudier la réalité de ce «feuilletage » nous avons relevé pour chacun des articles du corpus le nombre de références à des travaux antérieurs, que ces travaux soient du même auteur ou dus à d'autres historiens, ensuite le nombre de citations de passages d'ouvrages ou d'articles de ces mêmes historiens sans tenir compte toutefois de citations qui n'auraient été que d'un seul mot ou d'une seule expression, puis les références directes à des sources primaires, documents d'époque ou parfois rééditions de documents d'époque ${ }^{7}$ et enfin les citations extraites de sources primaires.

Les résultats peuvent être consultés dans le tableau 1. Afin d'en améliorer la lisibilité, les cases ne présentant aucune occurrence ont volontairement été laissées vides.

Il est facile, à la lecture de ce tableau, de constater la véracité de la citation de Prost quant au "feuilletage » du texte historique, même si la proportion de citations de sources primaires par rapport aux citations d'historiens est inversée (trois occurrences de sources primaires contre neuf occurrences de textes empruntés aux historiens). Une étude statistique menée sur un plus grand nombre de textes permettrait de vérifier s'il s'agit là d'un hasard ou d'une différence entre l'écriture de l'histoire en français, ce dont parlait Prost, et l'écriture de l'histoire en anglais.

Tableau 1. Nombre de références aux travaux antérieurs

\begin{tabular}{|l|c|c|c|c|c|}
\hline Auteurs & $\begin{array}{c}\text { Nombre } \\
\text { de pages }\end{array}$ & $\begin{array}{c}\text { Références aux } \\
\text { travaux } \\
\text { antérieurs }\end{array}$ & $\begin{array}{c}\text { Citations de } \\
\text { travaux } \\
\text { antérieurs }\end{array}$ & $\begin{array}{c}\text { Références aux } \\
\text { sources } \\
\text { primaires }\end{array}$ & $\begin{array}{c}\text { Citations de } \\
\text { sources } \\
\text { primaires }\end{array}$ \\
\hline ASH & 10 & & & 15 & \\
\hline BACHRACH & 21 & 80 & & 35 & \\
\hline
\end{tabular}




\begin{tabular}{|c|c|c|c|c|c|}
\hline BILLINGTON & 13 & 7 & & 23 & 14 \\
\hline DYHOUSE & 19 & 4 & & 7 & \\
\hline FINDLAY & 14 & 31 & 13 & & \\
\hline HERDER & 11 & & 7 & 41 & \\
\hline HOBSBAWM & 45 & 93 & 3 & & 4 \\
\hline LEE & 25 & 97 & 1 & 25 & \\
\hline McCULLOUGH & 6 & 23 & 2 & & \\
\hline McISAAC & 16 & 64 & 7 & 5 & \\
\hline $\begin{array}{l}\text { MORGAN- } \\
\text { JAMES }\end{array}$ & 10 & 2 & 14 & 2 & \\
\hline SIBLEY & 11 & 14 & 1 & 17 & 12 \\
\hline SUMMERS & 11 & & & 12 & \\
\hline SWAIN & 9 & 18 & 12 & 7 & \\
\hline $\begin{array}{l}\text { TREVOR- } \\
\text { ROPER }\end{array}$ & 20 & & & 43 & \\
\hline WERTHEIMER & 17 & 32 & & 37 & \\
\hline
\end{tabular}

Il faut à nouveau remarquer la faiblesse quantitative, voire parfois l'absence totale, de citations empruntées directement à d'autres historiens. Le même phénomène est encore plus vrai pour les citations directes de sources primaires, puisqu'elles ne figurent que chez trois auteurs. Toutefois, les références aux travaux antérieurs ou aux sources primaires étant par ailleurs quasi systématiques, il s'agit là sans doute dans ce cas d'un désir de simplification stylistique. Mais ce n'est là qu'une hypothèse : afin de tenir compte des différents paramètres entrant en jeu dans l'écriture, il faudrait dans un premier temps analyser le sujet de chacun des articles, son objectif, sa nouveauté par rapport aux écrits antérieurs éventuels, puis par la suite élargir la recherche en étudiant d'autres articles rédigés par le même auteur, d'autres articles publiés par des auteurs différents dans une même revue et enfin des articles portant sur le même sujet mais publiés dans des revues différentes avant de pouvoir affiner une conclusion acceptable. Ce n'était pas l'objectif de la présente étude.

\section{Un lien avec le présent}

61 Sans le temps il n'est pas d'histoire. "Le temps est le matériau premier de l'historien » (Caron 2005). Et, quelques lignes plus loin, Caron ajoute : « l'écriture même de l'histoire, 
c'est-à-dire le passage au récit [...] est d'abord et avant tout un ordonnancement du temps ». est appliqué à une époque ancienne. C'est ainsi que l'on trouve chez Findlay (2005: 47) à propos de l'époque victorienne : "Whether or not one sees this phenomenon as a prolonged act of 'ethnic cleansing', a form of cultural genocide, it resulted indisputably in the destruction of Gaelic society». Certes ethnic cleansing est proposé entre guillemets et est donc parfaitement acceptable, mais genocide est certainement à la limite de l'anachronisme et en tous cas du contre-sens ${ }^{8}$.

\section{L'objectivité du texte}

La consultation du tableau 1 montre bien les écarts importants qui existent d'un auteur à l'autre vis-à-vis de l'utilisation des sources. Ces différences nous permettent d'envisager l'hypothèse d'une écriture de l'histoire qui serait caractéristique d'un auteur et non d'un domaine, d'une profession et d'un genre. Cette hypothèse a été avancée dès notre introduction avec l'énoncé d'une hésitation entre "anglais de l'histoire » et « anglais de l'historien » ou « anglais des historiens ». Voici ce que dit à ce sujet un historien universitaire français contemporain :

L'histoire est une science au sens où elle est un savoir contrôlé par des procédures de travail et par une communauté; l'historien n'est pas dans une situation d'isolement comparable à l'écrivain et il est évident que l'histoire a des relations 
historiques et qui demeurent très fortes avec la littérature. C'est sans doute la moins formalisée des sciences sociales, c'est sans doute l'une des sciences sociales sur laquelle l'écriture pèse le plus. On voit très bien que les grands historiens, et même aujourd'hui ceux qui ont le plus de talent, ont une écriture qui les distingue des petits spécialistes gris [...] Je crois qu'il ne faut pas avoir honte de cela. (Prochasson 2008)

L'écriture de l'historien peut en effet, malgré tous les efforts voulus en faveur de la nécessaire objectivité scientifique, porter une trace que l'on pourrait qualifier d'idéologique, comme par exemple dans les derniers mots de la citation suivante: « Nevertheless, the invention of tradition played an essential role in maintaining the republic, if only by safeguarding it against both socialism and the right. » (Hobsbawm $1983: 270$ ). Il faut toutefois remarquer à ce propos que Hobsbawm, historien mondialement connu et reconnu, a adhéré au parti communiste à l'âge de 19 ans et que, contrairement aux autres membres du Communist Party Historians Group, il n'a pas quitté le parti en 1956 pour protester contre l'invasion de la Hongrie par les troupes soviétiques. Son apparent rejet des extrêmes dans la citation proposée, qui représente une prise de position subjective, l'historien n'étant en principe pas celui qui juge (Bloch 1949: 70), pourrait donc en fait être considéré comme un remarquable effort d'objectivité de sa part.

D'autres exemples, bien que rares, seraient possibles pour illustrer la subjectivité autre que celle due à une idéologie politique; on peut citer les énoncés suivants :

- "This claim [...] then, as now, was an attack on the male dominated sexual exploitation of women" (Billington 1988 : 126). Il faut noter que l'article de Billington s'intitule The dominant values of Victorian feminism et que Rosamund Billington se laisse parfois emporter par une idéologie féministe ${ }^{9}$. Dans ce même article, Billington hésite entre spokesperson (116) puis spokeswomen (121) et enfin spokespeople (124). Le contexte de chaque occurrence ne justifiant pas la différence de terme, cette hésitation est effectivement très probablement due à une influence idéologique.

- «clan tartans now worn, with tribal enthusiasm, by supposed Scots from Texas to Tokyo " (TrevorRoper 1983 : 41) ou encore

Indeed the whole concept of a distinct Highland culture and tradition is a retrospective invention. Before the later years of the seventeenth century, the Highlanders of Scotland did not form a distinct people. They were simply the overflow of Ireland [...] Racially and culturally [Scotland] was a colony of Ireland. (Trevor-Roper $1983: 15$ )

Le fait que Trevor-Roper soit mentionné deux fois dans ce cadre n'est certainement pas anodin; un historien reconnu a en effet probablement davantage tendance à laisser libre cours à ses opinions alors qu'un membre plus récent de la communauté est très certainement plus attentif à veiller à la neutralité de ses propos tant qu'il n'aura pas fait ses preuves devant ses pairs.

71 Cette hypothèse ne signifie évidemment pas que l'historien ne doive pas proposer d'affirmations puisque son rôle consiste à créer un savoir, donc à apporter de nouvelles connaissances, mais il doit s'attacher pour ce faire à la plus grande prudence : «En bornant sa part d'assurance à doser le probable et l'improbable, la critique historique ne se distingue de la plupart des autres sciences du réel que par un échelonnement des degrés sans doute plus nuancé » (Bloch 1952 : 66). 


\section{Prudence, modalités et certitudes}

72 Cet «échelonnement des degrés » est perceptible dans l'ensemble du corpus et, à quelque niveau que ce soit, aussi bien donc dans l'introduction que dans le développement ou la conclusion, la gamme habituelle des modalités se retrouve, depuis le doute méthodique jusqu'à la certitude : l'hypothèse (There is some reason to think that... / ...[he] might have replied... / A close examination might reveal... / It would therefore seem at first sight... / ...or was it because...? / ...evidence would suggest that...) voisine avec l'incertitude (...it remains unclear... / ...it is not at all certain...). La quasi-certitude (We may thus conclude that... / ...there is little doubt that... / ...it may be more accurate to say... / It is unlikely that... / ...the implication is surely... / ...it seems highly likely.../ It seems reasonable to assume that...) laisse ensuite la place à la certitude (...unquestionably... / ...it resulted indisputably in... / ...they were far from being... / ...there is no reason to doubt... / ... which points overwhelmingly to one conclusion...).

\section{La présence de l'auteur}

L'historien est handicapé dans son travail scientifique par l'absence d'observation directe des phénomènes qu'il décrit et qu'il se doit d'expliquer dans leur enchaînement. Son matériau n'est constitué que de témoignages textuels, iconographiques ou autres qu'il doit interpréter et dont il doit se méfier. Dyhouse mentionne à ce sujet le «bias of [...] observers » (1978: 180). C'est là un écueil bien connu des historiens : « la poétique et la rhétorique du témoignage montrent qu'il n'y a pas de passage obligé de l'expérience à son récit » (Dornier 2004 : 405).

74 L'historien ne peut en effet se contenter de reproduire, traduire ou paraphraser, il doit créer son propre questionnement et tenter d'apporter des réponses. L'histoire s'oppose également aux autres sciences qui se fondent, entre autres, sur la reproductibilité des démonstrations. L'histoire ne peut reproduire les faits qui sont son objet d'étude. Cela pose évidemment aux historiens une difficulté toute particulière qui est celle de la frontière entre le réel et l'imaginaire :

Faits matériels, actes humains individuels et collectifs, faits psychiques, voilà tous les objets de la connaissance historique ; ils ne sont pas observés directement, ils sont tous imaginés. Les historiens - presque tous sans en avoir conscience et en croyant observer des réalités - n'opèrent jamais que sur des images. [...] Toute image historique contient donc une forte part de fantaisie. (Langlois \& Seignobos 1898 : 124-125)

Cette «fantaisie » pose le problème de la présence de l'auteur, présence qui peut être immédiatement visible.

Outre les exemples mentionnés supra dans le paragraphe consacré à l'objectivité du texte, on relève chez certains auteurs, et à nouveau en particulier chez Trevor-Roper et Hobsbawm, ce qui tend d'ailleurs à renforcer la conclusion déjà tirée quant aux écarts que peut se permettre un membre reconnu de l'institution, la présence du pronom personnel I (...as far as I know... / I know of no case of...) ou de l'adjectif possessif my (...by my count...).

77 Il est possible de considérer au même titre le we universitaire, qui n'est qu'un masque $\mathrm{du} I$ (...we have argued that... / ...if we take into consideration... / ...we should not be too critical of... / ...we can distinguish....). 
78 Dans notre corpus le «subjective I» que refusait Gogsik cité supra ne se trouve effectivement attesté que chez Trevor-Roper et Hobsbawm ${ }^{10}$. Chez les autres auteurs, il s'efface systématiquement devant we dont la fréquence d'occurrence n'est toutefois guère élevée, le passif étant privilégié.

79 L'auteur est donc parfois présent de manière visible, mais cette présence est relativement rare. La présence indirecte de l'auteur se fait plus volontiers sentir à partir d'une analyse stylistique.

80 Lorsque Hobsbawm (1983 : 287) utilise la litote suivante : «It is not unreasonable to suppose that the masses were unaware of it ", il y a bien un choix délibéré visant à susciter l'intérêt du lecteur. Par son choix d'écriture Hobsbawm choisit délibérément de s'écarter d'une norme.

81 Il faut également noter l'existence de traits humoristiques, en particulier chez TrevorRoper : "traditions which he had discovered more often, perhaps, in the officers' mess than in the straths and glens of Scotland ( (Trevor-Roper $1983: 28$ ), ou encore "Such was the effect, even in decorous Edinburgh, of the free circulation of wholesome air under the Highlander's kilt »(Trevor-Roper 1983 : 29).

82 Enfin, des métaphores ou images pimentent parfois le texte et nous les trouvons une nouvelle fois chez le même auteur: "The essential figures in this episode were two of the most seductive characters who have ever ridden the Celtic hobby-horse or aerial broomstick: the brothers Allen » (Trevor-Roper 1983 : 31).

83 Il y a donc bien des caractéristiques systématiquement présentes chez certains auteurs et qui justifient une nouvelle fois notre tendance initiale à retenir «anglais des historiens » comme appellation possible.

\section{L'auteur et le lecteur}

84 L'auteur de l'article de recherche présuppose un destinataire et, nous l'avons rappelé, ce destinataire doit selon l'expression de Sir John Plumb être « entertained».

85 L'historien s'adresse donc parfois assez directement à son lecteur par un conseil ou une forte suggestion :

- «The sheer effrontery of the Macphersons must excite admiration » (Trevor-Roper 1983 : 17),

• « it is important to stress» (Sibley $2004: 44)$,

- « it is curious to see... » (Findlay $2005: 53$ ),

- « it should be emphasized that... » (Dyhouse 1978 : 184).

Le destinataire peut aussi être inclus par un we qui n'est plus simplement un simple masque de $I$ mais bien un you and $I$ :

- « if we wish to understand why... » (Summers $1995: 124)$,

- « if we remember... » (Billington $1988: 117)$,

- « we know what it meant to a small number of... » (Sibley $2004: 42)$.

\section{Un regard réflexif sur la discipline}

Une dernière caractéristique du discours de l'histoire, tel qu'il apparaît dans notre corpus, est la réflexion qui peut être menée sur la discipline à l'intérieur même de la démonstration de la thèse des auteurs. Il arrive en effet que, par le biais d'une 
digression, l'historien s'interroge sur son propre domaine ou sur les contraintes de sa démarche :

- « However, history is not rational: or at least it is rational only in parts. » (Trevor-Roper 1983 : 24)

- «In economic history we often witness the ruin of the bold, imaginative, sometimes fantastic pioneer whose work is then taken over and carried to success by a more pedestrian entrepreneur. » (TrevorRoper $1983:$ 38)

- «Any historian has to some extent to try to discount the bias of [..] observers. » (Dyhouse 1978 : 180).

Cette démarche est, semble-t-il, couramment admise par les historiens :

L'histoire n'est pas une critique épistémologique. Elle reste un récit. Elle raconte son propre travail et, simultanément, le travail lisible dans un passé. Elle ne comprend d'ailleurs celui-ci qu'en élucidant sa propre activité productrice et, réciproquement, elle se comprend elle-même dans l'ensemble et la succession de productions dont elle est elle-même un effet. (de Certeau 1975 : 68)

Ce regard réflexif mérite d'être mentionné puisqu'il apparaît chez deux des dix-huit auteurs de notre corpus et qu'il est reconnu par les historiens eux-mêmes. Il s'agit là sans doute d'un trait caractéristique de la recherche dans le domaine des sciences humaines.

\section{Conclusion}

Ainsi que le titre l'annonçait, il ne s'agit là que d'une première ébauche de caractérisation de l'anglais de l'histoire, ne portant en outre que sur le genre que constitue l'article de recherche. L'étude de documents plus importants, les livres donc, devrait permettre d'affiner et ainsi confirmer ou d'infirmer ces remarques et hypothèses. En particulier, dans la mesure où l'article est un genre normé relativement récent, une étude diachronique pourrait montrer une évolution certaine du discours historique en général, répondant pour chaque époque aux attentes du lecteur contemporain. L'on aurait du mal de nos jours, par exemple, à imaginer un écrit universitaire contemporain dans lequel figureraient des énoncés d'une telle «incorrection politique »: «[...] an elaborate attempt to assure the Negro of equal rights in everyday affairs" (Nevins \& Commager 1942: 237), ou encore "Recent slaves, whose grandfathers had perhaps been African savages, who could not read a line of print... » (Nevins \& Commager $1942: 237)^{11}$.

91 Sur un plan comparatif et dans le cadre du discours historique en général, donc non plus simplement en anglais et uniquement dans le cadre d'articles de recherche, l'étude des historiens francophones permettrait selon toute probabilité de trouver un certain nombre de points communs. C'est ainsi que, si l'on considère simplement les choix stylistiques destinés à soutenir l'intérêt du lecteur, on appréciera à leur juste valeur les quelques énoncés suivants extraits d'un ouvrage de Bennassar qui rappellent sans aucun doute la prose de Trevor-Roper régulièrement citée supra: «Mais il n'en fallait pas davantage à l'irascible Henry VIII, déjà occupé de la manière dont il pourrait remplacer Anne par Jane Seymour, dans son lit et sur le trône " (2006:27) ou encore « la volcanique Marie » $(2006: 77)$.

Pour conclure sur cette ébauche de caractérisation enfin, il semble possible d'affirmer que la plupart des remarques énoncées pourraient fort probablement s'appliquer à l'ensemble connu sous l'appellation de « lettres et sciences humaines ", qu'il s'agisse de 
la structure de l'article, dont on a vu qu'elle rappelait très fortement tout ce qui a déjà été dit sur l'article de recherche en sciences, ou même qu'il s'agisse du discours luimême qui apparaît comme représentatif du discours de tout universitaire qui cherche à démontrer une thèse ou à expliquer une observation.

Toutefois, et sans vouloir oublier l'idée de "regard réflexif », la première observation proposée dans l'étude, à savoir l'absence de terminologie propre au domaine est sans doute le trait marquant, le trait distinctif du discours de l'histoire. Cette remarque est en effet à mettre en opposition avec l'affirmation de Swales selon laquelle " $a$ highly specialized terminology» $(1990: 29)$ est une des six caractéristiques essentielles d'une communauté de discours :

It is hard to conceive, at least in the contemporary English-speaking world, of a group of well-established members of a discourse community communicating among themselves on topics relevant to the goals of the community and not using lexical items puzzling to outsiders. It is hard to imagine attending perchance the convention of some group of which one is an outsider and understanding every word. If it were to happen - as might occur in the inaugural meeting of some quite new grouping - then that grouping would not yet constitute a discourse community. (1990: 26-27)

Serait-ce à dire alors que les historiens ne constituent pas une communauté de discours?

Je tiens à remercier ici André Béziat, Isabelle Cases, Aymat Catafau, Mireille Courrent, Nicolas Marty et Blandine Pennec, mes collègues anglicistes, hellénistes et historiens, qui ont bien voulu m'aider à élucider les points les plus ambigus de mon corpus. Aucune erreur ou interprétation erronée subsistante ne saurait leur être imputée.

\section{BIBLIOGRAPHIE}

\section{Références du corpus}

Ash, Marinell. 1993. « William Wallace and Robert the Bruce: the life and death of a national myth ». In Samuel R. et P. Thompson (dir.), The Myths we Live by. Londres : Routledge, 83-94.

Bachrach, David S. 2003. « The organisation of military religion in the armies of King Edward I of England (1272-1307)». Journal of Medieval History 29, 265-286.

Bennassar, Bartolomé. 2006. Le Lit, le pouvoir et la mort. Paris : Éditions du Fallois.

Billington, Rosamund. 1988. « The dominant values of Victorian Feminism ». In Sigsworth E. M. (dir.), In Search of Victorian Values. Manchester : Manchester University Press, 116-130.

Dyhouse, Carol. 1978. « The role of women: from self-sacrifice to self-awareness ». In Lerner L. (dir.), The Victorians. Londres : Methuen.

Findlay, William. 2005 « 'Almost afraid to know itself?' The unfolding of Scottish identity in Victorian times ». In Victorian Paradoxes, Revue du GRAAT 30, Tours, Presses universitaires de Tours. 
Herder, Michelle. 2005. « Substitute or subordinate? The role of a male procurator at a Benedictine women's monastery ». Journal of Medieval History 31, 231-242.

Hobsbawm, Eric. 1983. « Mass-producing traditions: Europe, 1870-1914 ». In Hobsbawm E. et T. Ranger (dir.), The Invention of Tradition. Cambridge : Cambridge University Press.

Lee, Robert. 2005. « Domestic service and female domestic servants: A port-city comparison of Bremen and Liverpool, 1850-1914». The History of the Family 10, 435-460.

McCullogh, John et al. 2006. «Culling the cousins: Kingship, kinship, and competition in midmillenial England ». The History of the Family 11, 59-66.

McIsaac Cooper, Sheila. 2005. « Service to Servitude? The decline and demise of life-cycle service in England ». The History of the Family 10, 367-386.

Morgan-James, Annie. 2001. « Myth and reality: filmic images of Scotland ». Revue française de civilisation britannique 11/2, Le Cinéma britannique, Paris, Presses de la Sorbonne Nouvelle, 29-40.

Nevins, A. et H. Commager. 1942. A Pocket History of the United States. New York : Washington Square Press.

Sibley, Richard. 2004. « Feminity or feminism? Women's sport and dress in Victorian Britain ».In Revue française de civilisation britannique 12/4, Constantes et évolutions de la société britannique XIX ${ }^{{ }^{-}}$ XXI ${ }^{e}$ siècles Paris, Presses de la Sorbonne Nouvelle, 37-47.

Swain, Shurlee. 2005. « Maids and mothers: Domestic servants and illegitimacy in 19th-century Australia ». The History of the Family 10, 461-471.

Summers, Anne. 1995. « Ministering angels: Victorian ladies and nursing reforms ». In Marsden G. (dir.), Victorian Values. Londres : Longman.

Trevor-Roper, Hugh. 1983. " The invention of tradition: The Highland tradition of Scotland ». In Hobsbawm E. et Ranger T. (dir), The Invention of Tradition. Cambridge : Cambridge University Press.

Wertheimer, Laura. 2005. « Illegitimate birth and the English clergy, 1198-1348 ». Journal of Medieval History 31, 211-229.

\section{Références bibliographiques}

Bachelard, Gaston. 1967 [1934] La formation de l'esprit scientifique. $5^{\mathrm{e}}$ éd. Paris : Librairie philosophique J. Vrin. CollectionBibliothèque des textes philosophiques.

Barthes, Roland. 1984. « Le Discours de l'histoire » In Le Bruissement de la langue. Paris : Éditions du Seuil.

Bloch, Marc. 1952. Apologie pour l'histoire ou métier d'historien, Cahiers des Annales 3. Paris : Armand Colin.

Boiste, Pierre. 1828. Dictionnaire universel de la langue française. Bruxelles : Frechet.

Bonnot, Gabriel, abbé de Mably, 1796. De la manière d'écrire l'histoire. <http://www.eliohs.unifi.it/ testi/700/mably/ecrire.html>. Consulté le 7 mai 2008.

Carney, Todd F. 1996. Guide to Writing History Papers. <http://www.sou.edu/history/ carney/ writing.htm>. Consulté le 10 juin 2008.

Caron, Jean-Claude. 2002. "Conclusion. Le temps des historiens ou regards sur le passé », Revue d'histoire du XIXe siècle, 25, mis en ligne le 29 juin 2005. <http://rh19.revues.org/index421.html>. Consulté le 20 novembre 2008. 
Coffin, Caroline. 2006. Historical Discourse: the language of time, cause and evaluation. Londres : Continuum.

Darbo-Peschanski, Catherine. 2007. L'Historia. Commencements grecs. Paris : Gallimard.

De Certeau, Michel. 1975. L'Écriture de l'histoire. Paris : Gallimard.

Deleplace, Marc. 2006. Analyse du discours : quand l'anarchie invite à la convergence. Epistémologie. <http://revolution-française.net/2006/02/04/24-analyse-du-discours-quand-lanarchie-invite-ala-convergence>. Consulté le 10 juin 2008.

Dornier, Carole. 2004. « Récit factuel et littérarité ». In Amossy, Ruth et Dominique Maingueneau(dir.), L'Analyse du discours dans les études littéraires. Toulouse : Presses Universitaires du Mirail, 405-416.

Foucault, Michel, 1969. L'Archéologie du savoir. Paris : Gallimard.

Giroud, Jean-Claude. 1979. « Apologie pour l'historien ». In Greimas, Algirdas Julien et Eric Landowski (dir.), Introduction à l'analyse du discours en sciences sociales. Paris : Hachette, 129-139.

Gogsik, Karen. 2005. Writing the History Paper, Challenges of Writing History. <http:// www.dartmouth.edu/ writing/materials/student/soc_sciences/history.shtml>. Consulté le 10 juin 2008.

Guereña, Jean-Louis. 2007. «Civilisationnistes ou historiens?». Cahiers de civilisation espagnole contemporaine 1, Mis en ligne le 13 juin 2007. <http://ccec.revues. org/index117.html>. Consulté le 02 février 2009.

Langlois, Charles-Victor et Charles Seignobos. 1898. Introduction aux études historiques. Paris : Hachette.

Lucien de Samosate. 1912. « Comment il faut écrire l'histoire », Euvres complètes, Trad. Eugène Talbot. Paris : Hachette. <http://remacle.org/bloodwolf/philosophes/Lucien /histoire.htm>. Consulté le 14 juin 2008.

Minc, Alain. 2008. « La France a manqué son destin marchand ». Le Figaro, 19 septembre.

Petit, Michel. 2004. «Quelques réflexions sur la fiction à substrat professionnel : du général au particulier ». In Petit, Michel et Shaeda Isani (dir.), Aspects de la fiction à substrat professionnel. Collection Travaux 2025. Bordeaux : Université Victor Segalen Bordeaux 2, 3-23.

Prochasson, Christophe. 2008. Entretien avec Emmanuel Laurentin. La fabrique de l'histoire. France Culture, Paris. 25 janvier.

Prost, Antoine. 1996. Douze leçons sur l'histoire. Paris : Éditions du Seuil.

Quéré, Henri. 2001. «Les dialogues de l'histoire : énonciation historique et paradoxe de l'historien ", Revue française de civilisation britannique, Mélanges en l'honneur de Roland Marx, Hors série $n^{\circ} 1$.

Swales, John. 1990. Genre Analysis English in Academic and Research Settings. Cambridge : Cambridge University Press.

Veyne, Paul. 1970. Comment on écrit l'histoire : essai d'épistémologie. Paris : Éditions du Seuil.

Schama, Simon. 2002. A History of Britain. Age-of-the-sage.org http://www.age-of-the-sage.org/ history/historian/Simon_Schama.html. Consulté le 20 janvier 2008. 


\section{ANNEXES}

Guide to Writing History Papers <http://www.sou.edu/history/ carney/writing.htm>

[...] The purpose of the introduction is to present your topic to your readers and then come to the point (your thesis). An introduction begins with a general statement concerning your topic and, with each succeeding sentence, focuses in on the thesis. [...]

In the main body of the essay you will present your argument in detail, and lay out your evidence for your reader. No rigid structural rules exist on how to develop the main body, but here are a few guidelines.

(1) Begin by making concessions to any possible opposing points of view. This suggestion relates to the psychology of argument. Admit when your opponent is correct and you weaken his arguments. No one understands why this is so, it just is.

(2) Place points of your argument in causal order, if you can find one. In history, as in life in general, one thing usually leads to another. [...]

(3) Group similar points together. If you can find no causal relationships, organize your points so you deal with closely related ideas together in the essay.

(4) Save your best point for last. Again, this relates to the psychology of argument. [...] The last point your readers encounter will be the one they remember best - make it your most powerful one.

(5) As you develop the main body of your essay, make the last sentence (or the last idea) of each paragraph lead naturally into the first sentence (or first idea) of the next paragraph.[...] Paragraphs beginning with "However," or "Moreover," or "On the other hand," or with other such devices, let the reader know you are about to qualify what you have just said with new information, or elaborate on what you have already outlined, or look at the subject from a slightly different point of view. [...]

The concluding paragraph of your essay is almost as important as the introduction. It is here you relate your thesis and main points back to the general world. To do so, simply reverse the narrowing process used to construct the introduction. You will want to restate your thesis early in the conclusion, and often using the very same words helps to tie the entire essay together in the mind of the reader. You can also use the conclusion to make personal points about your topic that, for logical reasons, you left out of the main body. [...]

\section{NOTES}

1. Sans doute faudrait-il plutôt écrire le calame.

2. Nicolas Marty, communication privée

3. Des travaux sont pourtant parfois menés à de tels niveaux. On citera les articles de Coffin et en particulier son ouvrage Historical Discourse: the language of time, cause and evaluation (2006) dont le titre semble au premier abord attirant pour nous, mais qui s'intéresse prioritairement aux productions d'élèves du secondaire et qui se situe donc totalement en dehors de nos préoccupations.

4. Peut-être sous forme de boutade d'ailleurs : «Le jour venu, n'hésitez pas, braconnez sur les terres des historiens, ils en ont besoin!» 
5. Il faut toutefois noter qu'une telle conclusion aussi bien normée est parfois absente, l'article se terminant dans ce cas par quelques généralités.

6. Il s'agit de la croisade des Albigeois.

7. Il peut dans ces deux cas s'agir aussi bien de textes écrits que de documents iconographiques.

8. Genocide a en effet été défini en 1944 par Raphael Lemkin et l'on sait que l'utilisation qui en est faite à l'heure actuelle pour des raisons politiques est extrêmement sujette à caution.

9. Même si une telle prise de position est compréhensible dans le contexte de l'étude, le résultat est tout de même inattendu dans un article scientifique.

10. Il faut au passage noter que le $I$ est en revanche très fréquent dans les communications orales d'historiens britanniques et américains. Celles-ci ne font pas l'objet de cette étude mais elles ont été observées lors de colloques auxquels l'auteur a assisté lors de la préparation de son sujet.

11. Il faut toutefois mentionner que ces deux dernières citations sont extraites d'un livre et non d'un article, ce qui explique peut-être une plus grande liberté d'expression.

\section{RÉSUMÉS}

L'objectif de cet article est d'examiner les caractéristiques de l'anglais de l'histoire tel qu'il est possible de l'étudier à partir de seize articles de recherche en provenance de sources différentes et publiés au cours de ces trente dernières années. Il est également fait appel aux nombreux travaux d'historiens ayant réfléchi à l'écriture de leur discipline. Bien que retrouvant de nombreux éléments déjà présents dans les articles de recherche, et particulièrement rappelés par la communauté LANSAD au niveau de la structure et de l'organisation du discours, l'analyse suggère tout particulièrement l'absence d'une terminologie spécifique à la discipline " histoire ", mettant ainsi en évidence le caractère original de l'anglais de cette discipline.

This article examines the characteristics of the English of history as it appears in sixteen research articles of different origins published over the last thirty years. The study is also based on the many comments proposed by historians on the writing of their own discipline. Although it points out several constituting elements already present in other research articles, especially as described by the ESP community as far as the structure of discourse is concerned, the major conclusion suggested by the study is the absence of a specific terminology pertaining to history as a discipline, thus placing the English of history on an original footing when compared to the usual characteristics of ESP.

\section{INDEX}

Mots-clés : article de recherche, communauté linguistique, discours spécifique à un domaine, genre, histoire, terminologie

Keywords : domain-specific discourse, genre, history, language community, research article, terminology 


\section{AUTEUR}

\section{JEAN-LOUIS TROUILLON}

Jean-Louis Trouillon est maître de conférences HDR à l'Université de Perpignan Via Domitia. Ses enseignements LANSAD actuels s'adressent aux étudiants d'histoire, d'histoire de l'art et de géographie. Il intervient également en Master FLE dans le cadre de cours sur les TICE et l'apprentissage des langues ainsi que sur l'enseignement des langues de spécialité. Il est l'auteur ou co-auteur de nombreux didacticiels d'anglais scientifique, anglais militaire et anglais général. Il est aussi l'auteur d'un Dictionnaire bilingue de géopolitique et relations internationales (Presses universitaires de Perpignan, 2006). Il est actuellement rattaché au LACES, EA 4140. jeanlouis.trouillon@wanadoo.fr 